\section{Interleukin 4 promotes phagocytosis of murine leukemia cells counteracted by CD47 upregulation}

\author{
Pablo Peña-Martínez, Ramprasad Ramakrishnan, Carl Högberg, \\ Caroline Jansson, David Gisselsson Nord and Marcus Järås
}

Division of Clinical Genetics, Department of Laboratory Medicine, Lund University, Lund, Sweden

Haematologica 2022

Volume 107(4):816-824

\section{Correspondence:}

MARCUS JÄRÅS

marcus.jaras@med.lu.se

Received: August 25, 2020.

Accepted: April 27, 2021.

Pre-published: May 6, 2021.

https://doi.org/10.3324/haematol.2020.270421

(C)2022 Ferrata Storti Foundation

Material published in Haematologica is covered by copyright. All rights are reserved to the Ferrata Storti Foundation. Use of published material is allowed under the following terms and conditions:

https://creativecommons.org/licenses/by-nc/4.0/legalcode. Copies of published material are allowed for personal or internal use. Sharing published material for non-commercial purposes is subject to the following conditions:

https://creativecommons.org/licenses/by-nc/4.0/legalcode, sect. 3. Reproducing and sharing published material for commercial purposes is not allowed without permission in writing from the publisher.

\section{ABSTRACT}

ytokines are key regulators of tumor immune surveillance by controlling immune cell activity. Here, we investigated whether interleukin 4 (IL4) has antileukemic activity via immune-mediated mechanisms in an in vivo murine model of acute myeloid leukemia driven by the $M L L-A F 9$ fusion gene. Although IL4 strongly inhibited leukemia development in immunocompetent mice, the effect was diminished in immune-deficient recipient mice, demonstrating that the antileukemic effect of IL4 in vivo is dependent on the host immune system. Using flow cytometric analysis and immunohistochemistry, we revealed that the antileukemic effect of IL 4 coincided with an expansion of $\mathrm{F} 4 / 80^{+}$macrophages in the bone marrow and spleen. To elucidate whether this macrophage expansion was responsible for the antileukemic effect, we depleted macrophages in vivo with clodronate liposomes. Macrophage depletion eliminated the antileukemic effect of IL4, showing that macrophages mediated the IL4-induced killing of leukemia cells. In addition, IL 4 enhanced murine macrophage-mediated phagocytosis of leukemia cells in vitro. Global transcriptomic analysis of macrophages revealed an enrichment of signatures associated with alternatively activated macrophages and increased phagocytosis upon IL4 stimulation. Notably, IL4 concurrently induced Stat6-dependent upregulation of CD47 on leukemia cells, which suppressed macrophage activity. Consistent with this finding, combining CD47 blockade with IL4 stimulation enhanced macrophage-mediated phagocytosis of leukemia cells. Thus, IL4 has two counteracting roles in regulating phagocytosis in mice; enhancing macrophage-mediated killing of leukemia cells, but also inducing CD47 expression that protects target cells from excessive phagocytosis. Taken together, our data suggest that combined strategies that activate macrophages and block CD47 have therapeutic potential in acute myeloid leukemia.

\section{Introduction}

Acute myeloid leukemia (AML) is a fatal disease characterized by an accumulation of myeloid blasts in the bone marrow. For AML to develop, the malignant cells must escape tumor immune surveillance. Several evasion mechanisms have been described in AML, mainly associated with suppression of natural killer (NK) cells and macrophages..$^{1-3}$ Suppression of NK cells is mediated by secretion of ligands from the leukemic blasts and through direct cell-cell interactions with leukemic cells. ${ }^{4}$ An absence of NKG2D ligands on leukemia stem cells mediates their immune evasion. ${ }^{5}$ The main inhibitory signal to macrophages is CD47, which is upregulated on AML cells and protects them from phagocytosis. ${ }^{2}$ Paradoxically, tumor-associated macrophages in AML also contribute to immune suppression. ${ }^{6,7}$ Whereas interleukin (IL)2 and IL15 promote restoration of NK cell function in $\mathrm{AML},{ }^{8}$ anti-CD47 blocking antibodies can rescue macrophage function. ${ }^{9}$ Whether cytokine treatment can restore and boost macrophage-mediated antileukemic activity is currently unclear.

In a syngeneic murine AML model, we previously found that IL4 exerts 
antileukemic activity by inducing Statb-dependent apoptosis of AML cells. ${ }^{10}$ Elevated IL4 levels in mice eradicate AML cells in both the spleen and bone marrow, resulting in increased survival. Under physiological conditions, IL4 is a pleiotropic cytokine that regulates several immunological processes, such as B-cell class switching, T helper cell maturation, alternative activation of macrophages, and activation of NK cells. ${ }^{11,12}$ IL 4 can bind to the IL4 receptor (IL4R) type I receptor complex, a heterodimer of the IL4R alpha (IL4RA) and IL2 receptor subunit gamma (IL2RG) chains, or to the IL4R type II receptor complex, a dimer of IL4RA and IL13RA1. ${ }^{13}$ Whether immune cells also mediate the antileukemic activity of IL4 has not been previously explored.

In this study, we show that IL4 regulates phagocytosis by enhancing macrophage-mediated killing of AML cells and increasing CD47 expression on leukemia cells, which inhibits macrophages. Combined blockade of CD47 and IL4 stimulation enhanced macrophage-mediated killing of AML cells. Hence, our data suggest that combined strategies that activate macrophages and block CD47 have therapeutic potential in AML.

\section{Methods}

\section{The murine leukemia model}

All animal experiments were conducted according to the protocol approved by the Animal Care and Use Committee of the Lund/Malmö Ethical Committee. MLL-AF9 leukemias were generated in a dsRed C57BL/6 transgenic background (6051; Jackson Laboratory, Bar Harbour, NY, USA), as previously described. ${ }^{14}$ The $M L L-A F 9$ leukemia was serially propagated in sublethally irradiated (600 cGy) C57BL/6 recipient mice and leukemia stem cells were enriched as previously described.$^{15}$ All experiments involving murine leukemia cells were performed using tertiary or quaternary transplanted leukemia cells. As immunodeficient murine recipients, sublethally irradiated (250 cGy) NOD/SCID and NOD.Cg-

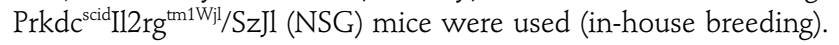
All mice used in experiments were age- and sex-matched.

\section{In vivo depletion of macrophages}

To deplete macrophages in mice transplanted with retrovirally transduced leukemia cells, we used intraperitoneal (i.p.) injection of $200 \mu \mathrm{L}$ of clodronate liposomes $(5 \mathrm{mg} / \mathrm{mL}$; Liposoma B.V., Amsterdam, the Netherlands). Controls were injected with phosphate-buffered saline. We administered the first injection of clodronate liposomes 1 day before injections of leukemia cells and repeated the procedure every tenth day. All mice in the survival experiments were sacrificed based on at least one of the following criteria: immobility, hunched back, hind leg paralysis, or dehydration.

\section{Phagocytosis assay}

For mouse phagocytosis assays, c-Kit ${ }^{+} \mathrm{dsRed}^{+}$murine $M L L-A F 9$ leukemia cells were added to macrophage cultures in a 2:1 ratio. After $18 \mathrm{~h}$, the cells were stained with a BV421-F4/80 antibody (BioLegend, San Diego, CA, USA), and the percentage of $\mathrm{F} 4 / 80^{+} \mathrm{dsRed}^{+}$cells was determined by FACS analysis.

For the CD47 blocking experiments, we incubated c-Kit ${ }^{+} \mathrm{dsRed}^{+}$ murine MLL-AF9 leukemia cells for $30 \mathrm{~min}$ with an anti-CD47 antibody or rat IgG2a isotype control $(30 \mu \mathrm{g} / \mathrm{mL}$; BioXCell, Lebanon, NH, USA), before co-culture with macrophages for $1 \mathrm{~h}$ at $37^{\circ} \mathrm{C}$. The percentage of $\mathrm{F} 4 / 80^{+} \mathrm{dsRed}{ }^{+}$cells was determined by flow cytometry as described above.
For human phagocytosis assays, we labeled human leukemia cell lines with the PKH67 green fluorescent cell dye according to the manufacturer's instructions (Sigma-Aldrich, Darmstadt, Germany) and stained macrophages with the PKH26 red fluorescent cell dye (Sigma-Aldrich). AML cells were mixed with human macrophages in a 2:1 ratio and incubated for either $2 \mathrm{~h}$ (Mono Mac 6 cells) or $18 \mathrm{~h}$ (MA9:16 cells). The percentage of $\mathrm{PKH}_{2} 6^{+}$ $\mathrm{PKH} 67^{+}$macrophages was determined by FACS.

\section{RNA sequencing analysis}

Global gene expression profiling was performed on sorted $\mathrm{F} 4 / 80^{+}$spleen cells from mice transplanted with IL4-overexpressing leukemia cells and non-transplanted irradiated controls. Cells were collected 12 days after irradiation. In addition, RNA sequencing was performed on macrophages produced in vitro by stimulating murine monocytes for 7 days with murine (m)CSF1 (25 $\mathrm{ng} / \mathrm{mL})$ and mIL4 $(20 \mathrm{ng} / \mathrm{mL})$ or only mCSF1.

Raw data and normalized gene expression data are available in the Gene Expression Omnibus database under accession number GSE155048.

\section{Results}

\section{The antileukemic activity of interleukin 4 in vivo is predominantly mediated via immune cells}

To determine whether immune cells contribute to the previously described antileukemic effects of IL 4 in vivo, ${ }^{10}$ we used a murine AML model driven by the MLL-AF9 (KMT2A-MLLT3) fusion gene. ${ }^{14}$ The leukemia cells were generated in a dsRed ${ }^{+}$transgenic background, allowing for convenient tracking of leukemia cells upon serial transplantations. ${ }^{16,17}$ Serial passaging of leukemia cells in mice did not alter IL4RA expression on AML blasts (Online Supplementary Figure S1A). Consistent with previous results, ${ }^{10}$ we confirmed that elevated IL4 levels mediated by retroviral expression in c-Kit ${ }^{+}$AML cells transplanted into mice (IL4 group) resulted in strong in vivo antileukemic activity. The IL4 group showed prolonged survival compared to controls and had almost no leukemia cells in the bone marrow or spleen at the time of sacrifice (Figure 1A, B, Online Supplementary Figure $S 1 B)$.

To address whether the antileukemic activity of IL 4 in vivo was immune-mediated, we used two strains of immunodeficient recipient mice: NOD/SCID mice, which lack $\mathrm{T}$ and $\mathrm{B}$ cells and have decreased activity of both NK cells and macrophages, ${ }^{18}$ and NSG mice, which additionally lack NK cells. ${ }^{19}$ In NOD/SCID animals, the antileukemic effect of IL 4 was reduced, and we observed increased levels of leukemia cells in the bone marrow and spleens compared to the levels in immunocompetent mice (Figure 1C, Online Supplementary Figure S1C). These findings suggest that immune cells at least partially mediate the antileukemic effect of IL4. To further characterize the antileukemic effect of IL4, we used the NSG mouse strain, which lacks a functional IL4 receptor type I complex because of deficiency in the $112 \mathrm{rg}$ gene. Of note, in NSG mice, the antileukemic effect of IL 4 was abolished, and survival was even shorter than in controls, with high levels of leukemia cells in both the bone marrow and spleens at the time of sacrifice (Figure 1D, Online Supplementary Figure S1D). These findings suggest that the antileukemic effect of IL 4 in vivo depends on immune cells expressing the IL4 receptor type I complex. 
A

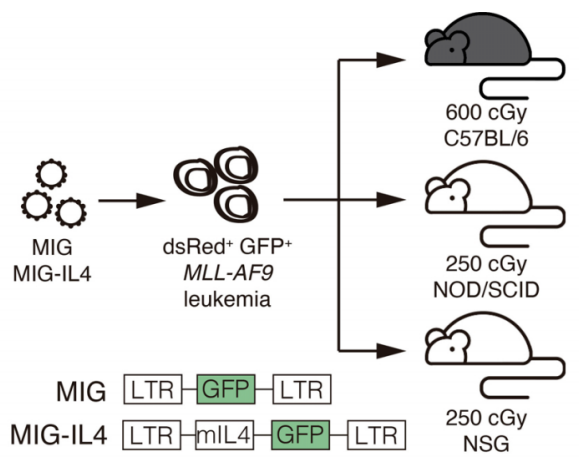

C

Survival NOD/SCID

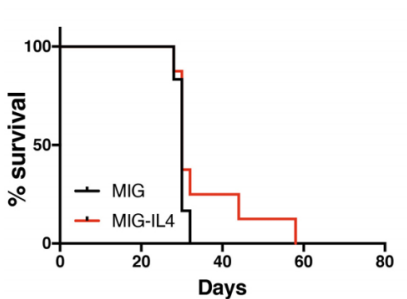

BM NOD/SCID

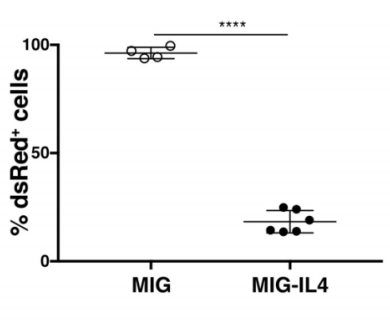

B

Survival C57BL/6J

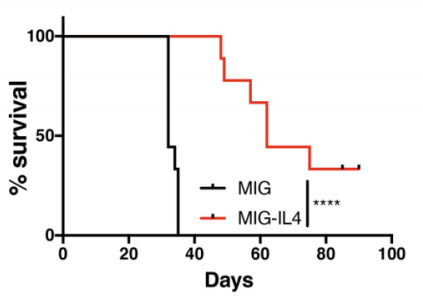

D

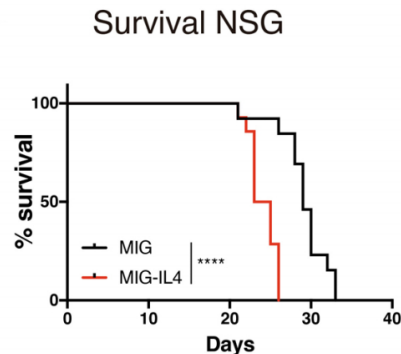

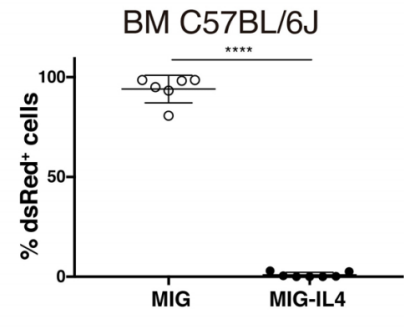

BM NSG

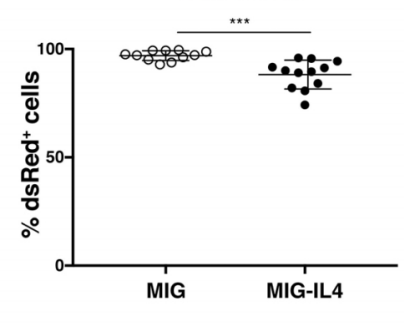

Figure 1. Interleukin-4 has antileukemic activity in a microenvironment-dependent manner. (A) dsRed ${ }^{+} \mathrm{c}-\mathrm{Kit}^{+} \mathrm{MLL}-\mathrm{AF9}$ acute myeloid leukemia (AML) cells were transduced with retroviral vectors coexpressing green fluorescent protein (GFP) and a murine interleukin 4 cDNA (MIG-IL4) or an empty control vector (MIG). Two days later, sorted $\mathrm{GFP}^{+} \mathrm{AML}$ cells were transplanted into sublethally irradiated mice. (B) Transplantation of 10,000 leukemia cells into C57BL/6 mice. Kaplan-Meier survival curves ( 9 mice per group, pooled from 2 independent experiments), and percentage of leukemia (dsRed ${ }^{+}$) cells in the bone marrow (BM) of mice at the time of sacrifice. (C) Transplantation of 30,000 leukemia cells into NOD/SCID mice. Kaplan-Meier survival curves (6 mice per group) and percentage of leukemia cells in the BM of mice at the time of sacrifice. (D) Transplantation of 30,000 leukemia cells into NSG mice. Kaplan-Meier survival curves (14 mice per group, pooled from 2 independent experiments), and percentage of leukemia cells in the BM of mice at the time of sacrifice. $* * * P<0.001 ; * * * * P<0.0001$.

\section{Interleukin 4 expands macrophages in vivo}

To identify the type of immune cell that mediates the IL4-induced antileukemic effects, we analyzed the hematopoietic compartment in mice receiving IL4-secreting AML cells. At day 19 after transplantation, we detected no IL4-induced alterations in blood cell lineages by flow cytometry (Figure 2A). Moreover, at this time-point, we detected no circulating leukemia cells in the blood of mice in the IL4 group (Figure 2B). In contrast, at day 27 after transplantation, the white blood cell, red blood cell, and platelet counts in the IL4 group were reduced compared to those in controls that had not been injected with leukemia cells (Figure 2C, Online Supplementary Figure S2A, $B)$. Of note, at the time of sacrifice, when the mice had succumbed to disease (Figure 1B), there was significant expansion of $\mathrm{F} 4 / 80^{+}$macrophages in the bone marrow (on average, $2.4 \%$ vs. $1 \% ; P<0.001$ ) and spleens (on average, $7.9 \%$ vs. $1.3 \% ; P<0.0001$ ) of IL 4 mice (Figure $2 \mathrm{D}$, Online Supplementary Figure S2C, D). We confirmed this IL4-induced increase in the proportion of macrophages by immunohistochemistry (Figure 2E, Online Supplementary Figure S2E). We also confirmed IL4RA expression on the $\mathrm{F} 4 / 80^{+}$cells from both groups of mice, supporting that IL4 receptor signaling may directly stimulate macrophages in this model (Online Supplementary Figure S2F). Hematoxylin staining of sections revealed extramedullary hematopoiesis in the spleens of the IL4 mice, as indicated by a marked increase in megakaryocytes and altered spleen architecture with increased red pulp and decreased white pulp (Online Supplementary Figure S2G). In addition to a reduction in leukemia cells, the decrease in circulating white blood cells, increased extramedullary hematopoiesis, and hypocellular bone marrow indicated that the elevated IL4 levels resulted in bone marrow failure in these animals. By contrast, in NSG mice, the IL4 group exhibited high levels of leukemia cells in the bone marrow, similar to the levels in the MIG control group (Online Supplementary Figure S2H).

\section{Interleukin 4 stimulation increases murine macrophage-mediated phagocytosis of leukemia cells}

To assess whether the IL4-induced expansion of macrophages in vivo was responsible for the antileukemic activity of IL4, we depleted macrophages by intraperitoneal injections of clodronate liposomes ${ }^{20,21}$ followed by injection of IL4-secreting AML cells (Figure 3A). Efficient depletion of macrophages was observed in the spleen but not in the bone marrow (Figure 3B). Consistent with the macrophage depletion, we found a proportional increase of leukemia cells in the spleen of these mice (on average, $33 \%$ vs. $6 \% ; P<0.05$ ), but not in the bone marrow (Figure $3 C)$. In contrast, depletion of macrophages had no effect on the level of leukemia cells in the MIG control group (Online Supplementary Figure S3A, B). These findings suggest that macrophages mediate the IL4-induced killing of leukemia cells.

Because macrophages kill cells by phagocytosis, we next assessed whether IL4 stimulation results in increased macrophage-mediated phagocytosis of leukemia cells in culture. Murine monocytes isolated from bone marrow were differentiated into macrophages for 7 days by supplementation of the culture medium with CSF1 (Figure 3D). The addition of IL4 to the medium resulted in increased phagocytosis of leukemia cells, as evident by macrophage acquisition of dsRed fluorescence (Figure 3E, F). Consistent with a more activated state, the IL4-stimu- 
A

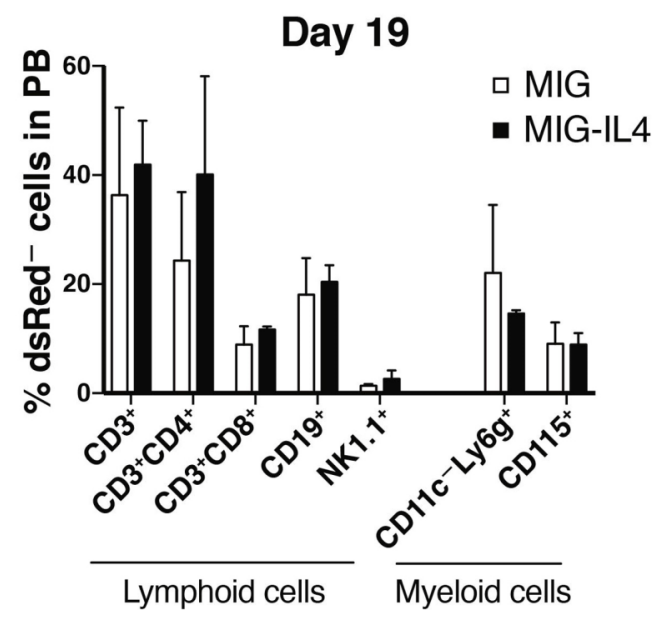

C

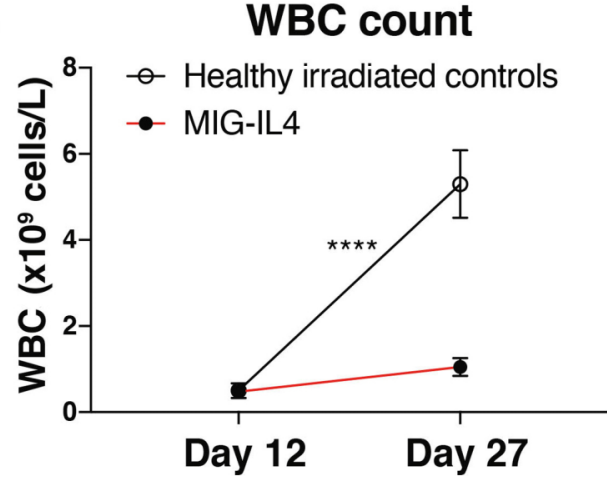

B

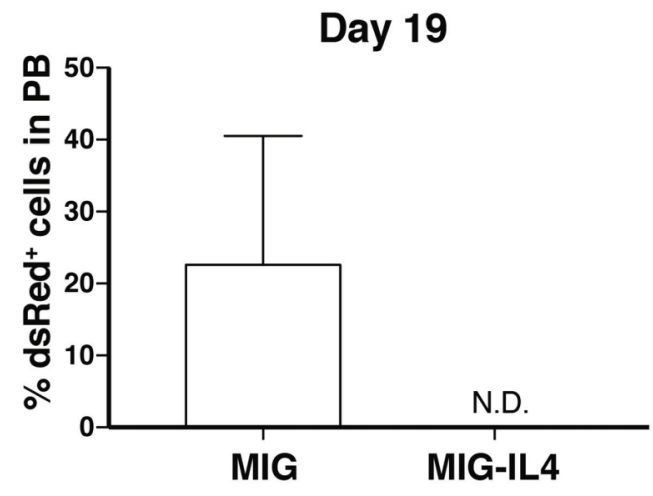

D

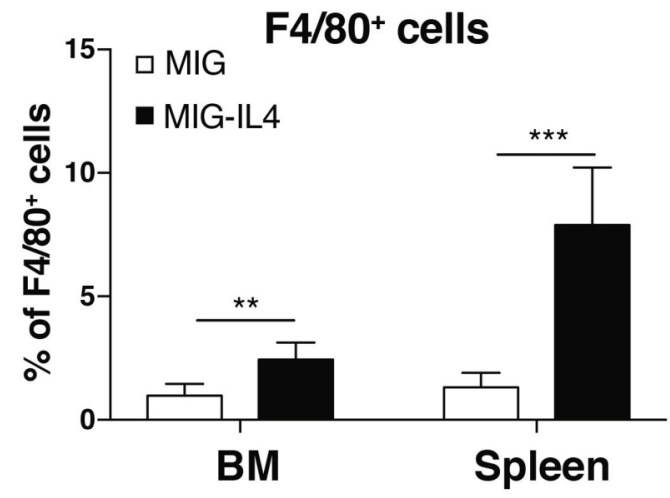

E

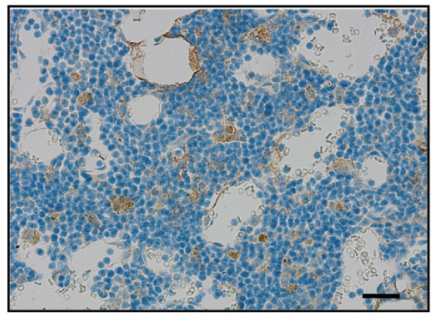

Healthy control

F4/80 IHC: BM

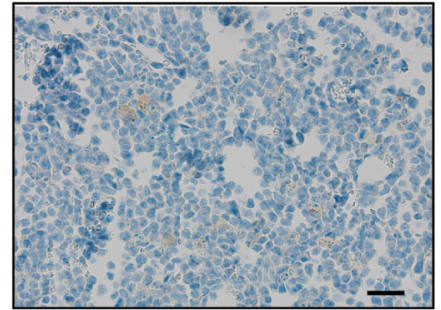

MIG

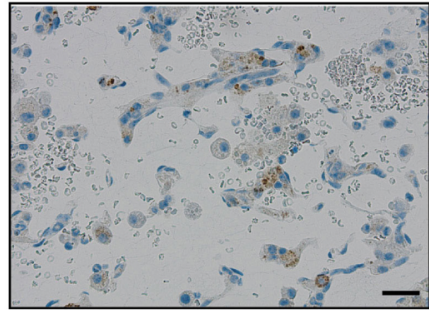

MIG-IL4

F4/80 IHC: Spleen

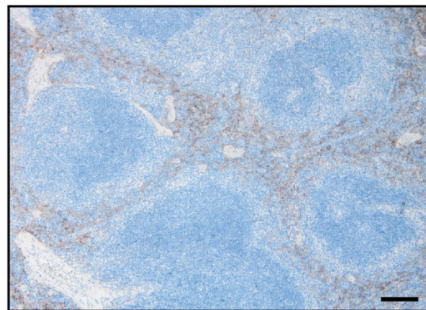

Healthy control

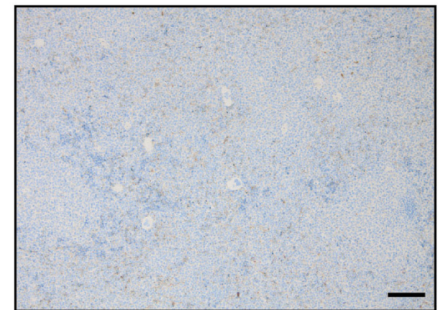

MIG

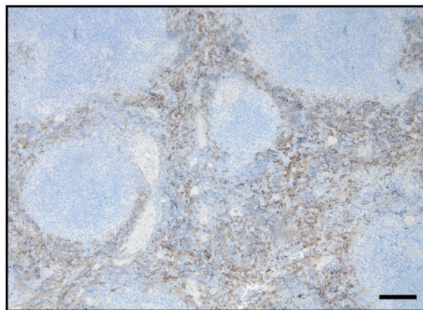

MIG-IL4

Figure 2. Interleukin 4 stimulation increases the frequency of macrophages in vivo. C57BL/ 6 mice were transplanted with 30,000 sorted green fluorescent protein $(\mathrm{GFP})^{+}$MLL-AF9 acute myeloid leukemia (AML) cells 2 days after transduction with retroviral vectors co-expressing GFP and a murine interleukin 4 cDNA (MIG-IL4) or a control vector (MIG). (A) Percentages of blood cell populations within dsRed cells 19 days after transplantation $(n=3)$. (B) Percentage of leukemia (dsRed ${ }^{+}$) cells in the peripheral blood on day 19 after transplantation $(n=3)$. (C) White blood cell counts at days 12 and 27 for MIG-IL4 and non-transplanted irradiated contro mice (IL4 group, $n=4$; controls, $n=3$ ). (D) Percentage of $F 4 / 80^{+}$cells within dsRed ${ }^{-}$cells in bone marrow and spleens of mice at the time of sacrifice (controls, $n=4$; IL4 group, $n=5)$. (E) Representative immunohistochemistry staining of $\mathrm{F} 4 / 80^{+}$cells in bone marrow (40x; scale bar, $\left.20 \mu \mathrm{m}\right)$ and spleens $(10 \mathrm{x}$; scale bar, $100 \mu \mathrm{m})$. BM: bone marrow; N.D.: not detected; PB: peripheral blood; WBC: white blood cell; IHC: immunohistochemistry. ${ }^{*} P<0.01$; ${ }^{*} * P<0.001$; ${ }^{*} * * * P<0.0001$. 
A

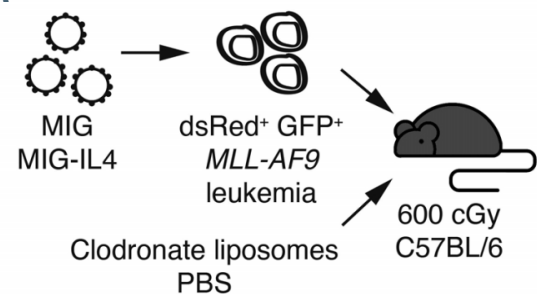

D

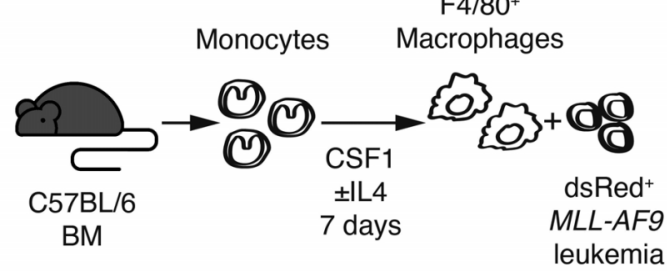

G

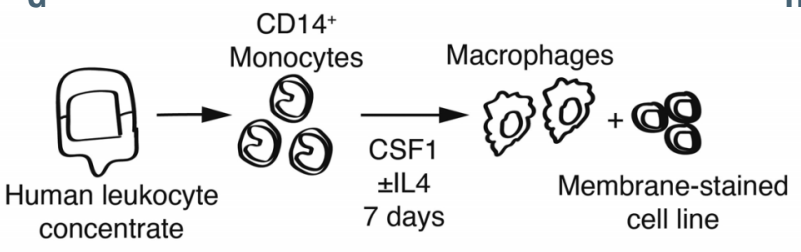

$\mathrm{H}$
$\mathrm{F} 4 / 80^{+}$cells

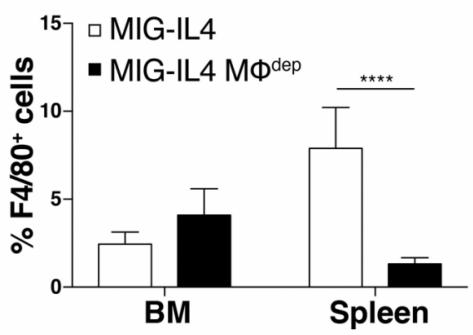

E
C
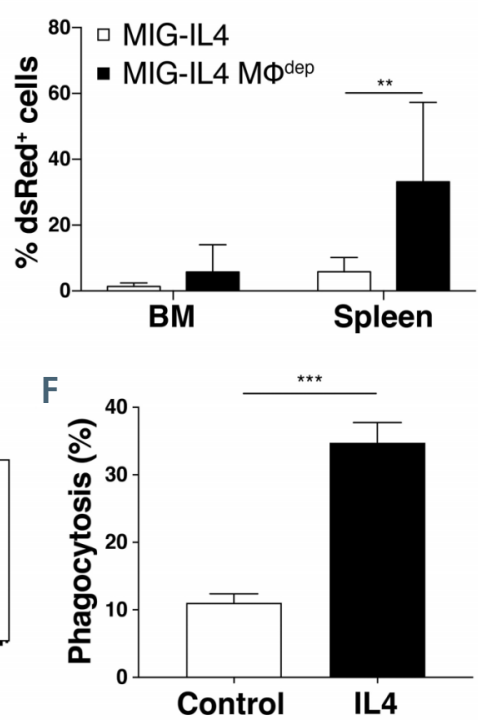

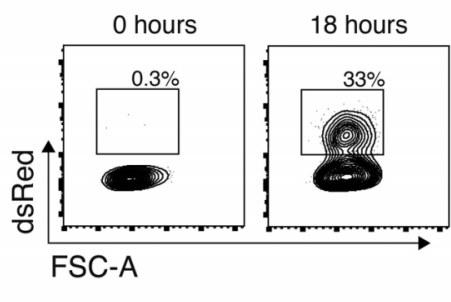

MA9:16 cell line

I

MM6 cell line
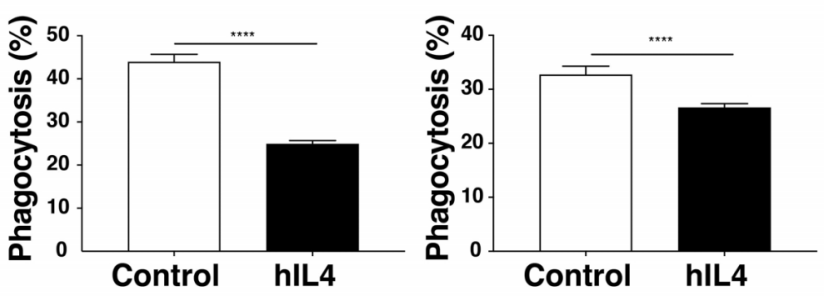

Figure 3. Interleukin 4 stimulation causes macrophage-mediated depletion of leukemia cells in vivo. (A) C57BL/ 6 mice were transplanted with 30,000 sorted green fluorescent protein (GFP) ${ }^{+}$MLL-AF9 acute myeloid leukemia (AML) cells transduced with retroviral vectors expressing a murine interleukin 4 cDNA (MIG-IL4) or GFP only (MIG; data presented in Online Supplementary Figure S2). One day prior to transplantation, mice received intraperitoneal (i.p.) injections of clodronate liposomes $\left(M \Phi^{\text {dep }}\right.$ group; $\left.n=4\right)$ or phosphate-buffered saline as control $(n=5)$. Every tenth day, new i.p. injections were performed. (B) Percentage of $F 4 / 80^{+}$cells and $(C)$ leukemia cells in bone marrow (BM) and spleens at the time of sacrifice in the IL4 group. (D) Monocytes were isolated from mouse BM and differentiated into macrophages in culture with mCSF1 $(25 \mathrm{ng} / \mathrm{mL})$ and $\mathrm{mlL} 4(20 \mathrm{ng} / \mathrm{mL})$ for 7 days, and then MLL-AF9 dsRed ${ }^{+}$AML cells were co-cultured with the macrophages. (E) Representative flow cytometry contour plots showing dsRed ${ }^{+}$cells within F4/80 cells in freshly mixed cultures $(0 \mathrm{~h})$ and after $18 \mathrm{~h}$ of co-culture with macrophages and dsRed ${ }^{+}$leukemia cells. (F) Phagocytosis assay with dsRed ${ }^{+}$AML cells and murine macrophages $(n=3)$. The percentage of dsRed ${ }^{+}$cells within $F 4 / 80^{+}$cells is presented. (G) CD14 cells were isolated from human blood and differentiated into macrophages in culture with human (h)CSF1 (25 ng/mL) and hIL4 (20 ng/mL) for 7 days and then co-cultured with membrane-stained AML cell lines. $(\mathrm{H})$ Phagocytosis assay with PKH67 ${ }^{+}$MA9:16 cells and PKH26 ${ }^{+}$human macrophages ( $\mathrm{n}=4$ ). The percentage of $\mathrm{PKH} 67^{+}$cells within $\mathrm{PKH} 26^{+}$cells is presented. (I) Phagocytosis assay with $\mathrm{PKH} 67^{+}$Mono Mac 6 cells and PKH26 ${ }^{+}$human macrophages ( $\mathrm{n}=5$ ). BM, bone marrow; MM6, Mono Mac 6; MФ, macrophage. $* * P<0.01 ; * * * P<0.001 ; * * * * P<0.0001$.

lated macrophages had an increased volume and were less irregular than unstimulated cells, as evaluated using phase holograph imaging (Online Supplementary Figure S4).

In contrast to its effect on murine macrophages, human IL4 is well known to differentiate human monocytes into anti-inflammatory macrophages. ${ }^{22}$ To assess how human IL4 affects phagocytosis of leukemia cells, human macrophages were stimulated with IL4 before mixing with AML cell lines. In line with a differential role of IL 4 in mice and humans, IL4 suppressed human macrophagemediated phagocytosis of the AML cells (Figure 3G-I).

\section{Interleukin 4 induces polarization of macrophages}

To investigate how IL4 affects the global gene expression of macrophages, we performed RNA sequencing of murine macrophages generated in vitro with or without IL 4 stimulation. In addition, we performed RNA sequencing on sorted $\mathrm{dsRed}^{-} \mathrm{F} 4 / 80^{+}$macrophages from mice transplanted with IL4-expressing leukemia cells and macrophages from leukemic control mice. In agreement with a described role for IL4 in promoting macrophage polarization, IL4 induced the expression of several genes associated with alternative activation of macrophages, including Arg1, Chil3, and Retnla (Figure 4A, Online Supplementary Figure $S 5 A, B),{ }^{22,23}$ which were among the most differentially upregulated genes (Online Supplementary Tables S1 and S2). Of note, IL4 also induced strong upregulation in vivo of the chemokine Ccl24, a biomarker for macrophages that originate from monocytes rather than tissue-resident macrophages (Figure 4A). ${ }^{24}$ Moreover, the IL4-induced macrophages showed downregulation of genes such as $C d 68$, which is associated with tumor-associated macrophages (Figure 4B), ${ }^{25}$ indicating that IL 4 differentiates macrophages into a phenotype that is distinct from tumor-associated macrophages.

We next performed gene set enrichment analysis to identify gene expression signatures enriched in the IL4-induced macrophages in vivo. In accordance with increased phagocytosis of macrophages stimulated with IL 4 in vitro, we found an enrichment of phagocytosis signatures in macrophages harvested from mice in the IL4 group (Figure 4C). Moreover, IL4 stimulation resulted in enrichment of genes 
A Differentially expressed genes
in vitro

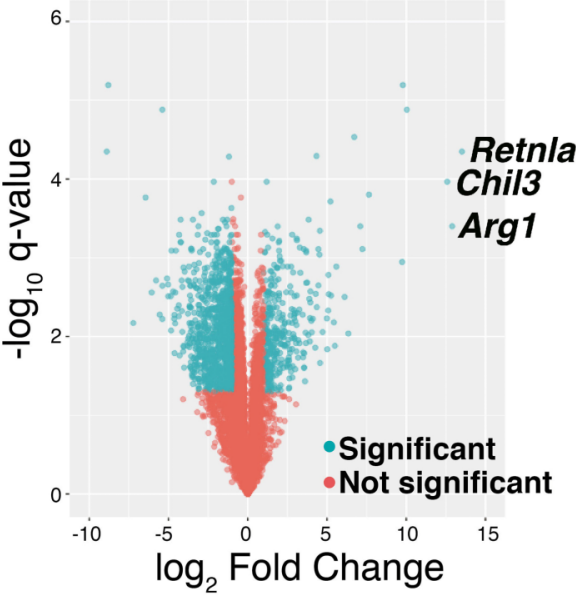

C

GO: Positive regulation of phagocytosis

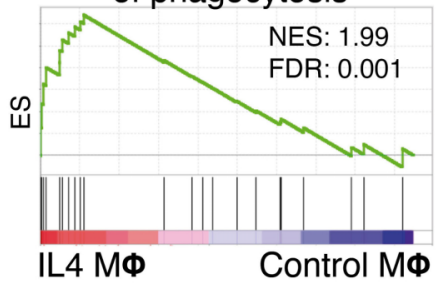

Differentially expressed genes in vivo

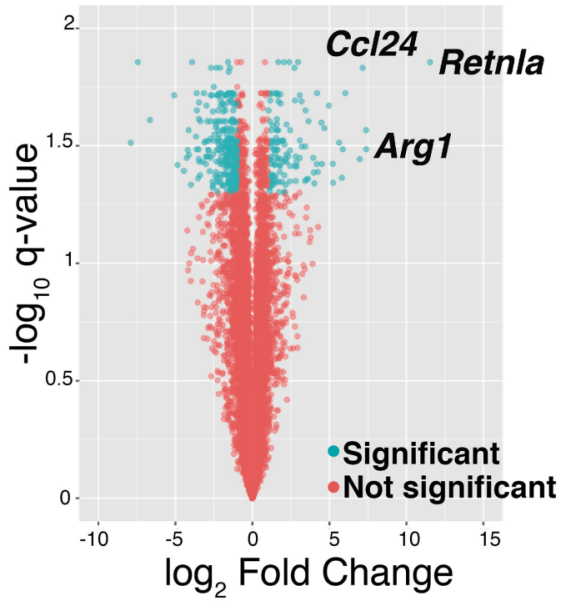

B

TAM markers in vivo

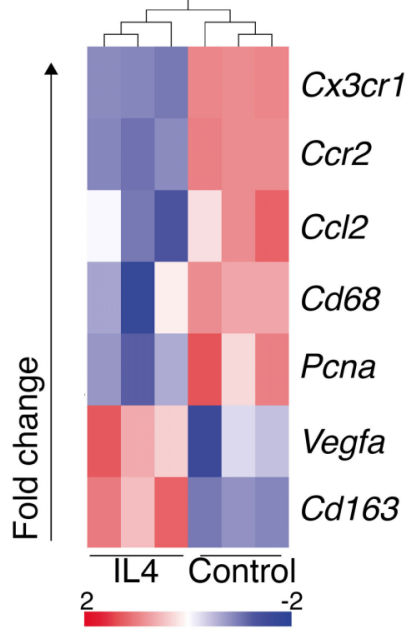

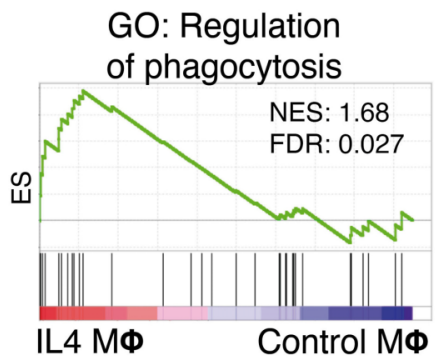

GO: MHC protein complex

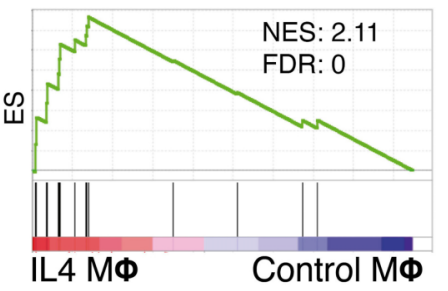

Figure 4. Interleukin 4 expands macrophages enriched for gene expression signatures associated with alternative activation of macrophages and phagocytosis. RNA sequencing was performed on murine macrophages generated from monocytes in vitro, and on sorted dsRed-F4/80+ macrophages from mice in the interleukin 4 (IL4) and control groups. (A) Volcano plots displaying differential gene expression between IL4-stimulated macrophages and control macrophages in vitro (left plot), and macrophages from mice in the IL4 or control group (right plot). The $y$-axis corresponds to the $-\log _{10}\left(\mathrm{q}\right.$-value) and the $x$-axis to the log ${ }_{2}$ of the gene expression fold change. Green dots represent significantly differentially expressed genes with a q-value $<0.05$ and fold change $>2.0$. (B) Heatmap showing expression of genes associated with upregulation in tumor-associated macrophages. IL4-stimulated macrophages and control macrophages were harvested from mice. (C) Gene set enrichment analysis revealed enrichment of phagocytosis and MHC protein complex signatures in macrophages harvested from mice. FDR, false discovery rate; GO: gene ontology; M $\Phi$, macrophage; NES, normalized enrichment score; TAM: tumor-associated macrophage.

associated with major histocompatibility complex (MHC) proteins (Figure 4C). To determine the influence of the in vivo microenvironment, we compared the gene expression profiles of IL4-stimulated macrophages generated in vitro and those generated in vivo (Online Supplementary Table S3). Macrophages generated in vivo exhibited a preferential upregulation of several markers associated with inflammation and immune activation (Online Supplementary Figure $S 5 C, D)$. Altogether, the gene expression data suggest that IL4 stimulation leads to an expansion of monocyte-derived macrophages with increased phagocytic activity.

\section{Interleukin 4 upregulates CD47 in a Stat6-dependent manner}

We next searched for IL4-induced mechanisms in leukemia cells that might affect their interactions with macrophages. Interestingly, the macrophage-inhibitory protein CD47 was upregulated on leukemia cells in the IL4 group compared to controls at the time of sacrifice (Figure 5A). Consistent with this finding, IL4 induced the expression of CD47 in leukemia cells in a dose-dependent manner, showing that IL 4 activates signaling that induces CD47 expression (Figure 5B). Moreover, according to RNA sequencing data that we had previously generated, ${ }^{10} \mathrm{Cd} 47$ was upregulated in both c-Kit ${ }^{+} \mathrm{AML}$ cells and normal c-Kit ${ }^{+}$bone marrow cells stimulated with IL4 (Figure 5C).

We next explored the mechanistic basis of the IL4induced upregulation of CD47. Because STAT6 is a critical downstream mediator of IL4R signaling, we used CRISPR/Cas9 genetic engineering to knock out Stat6 in Cas9-expressing MLL-AF9 AML cells using Stat6 sgRNA that we had previously characterized. ${ }^{10}$ Stat6 disruption hindered the IL4-induced upregulation of CD47 (Figure 5D), demonstrating that IL4 upregulates CD47 in a STAT6-dependent manner. Thus, in addition to activating murine macrophages, we identified a previously unknown role of IL 4 in protecting cells from phagocytosis via CD47 upregulation.

\section{Combined interleukin 4 treatment and CD47 blockade} results in enhanced macrophage-mediated phagocytosis of acute myeloid leukemia cells

Because CD47 protects cells from phagocytosis, we next evaluated whether the IL4-induced upregulation of CD47 on AML cells counteracts enhanced phagocytosis by IL4-stimulated macrophages. Consistent with this hypothesis, AML cells pre-treated for $24 \mathrm{~h}$ with IL4 and washed before co-culture with macrophages were partial- 
ly resistant to phagocytosis (Figure 5E). To overcome the inhibitory signal provided by increased CD47 expression, we used an $\alpha$-CD47 blocking antibody. Combined blocking of CD47 on AML cells and IL4 stimulation of macrophages resulted in enhanced phagocytosis of AML cells (Figure 5F). These findings show that IL4 has a dual role in murine phagocytosis by directly activating macrophages and enhancing their phagocytic activity, while also inducing CD47 expression that counteracts phagocytosis in target cells.

A
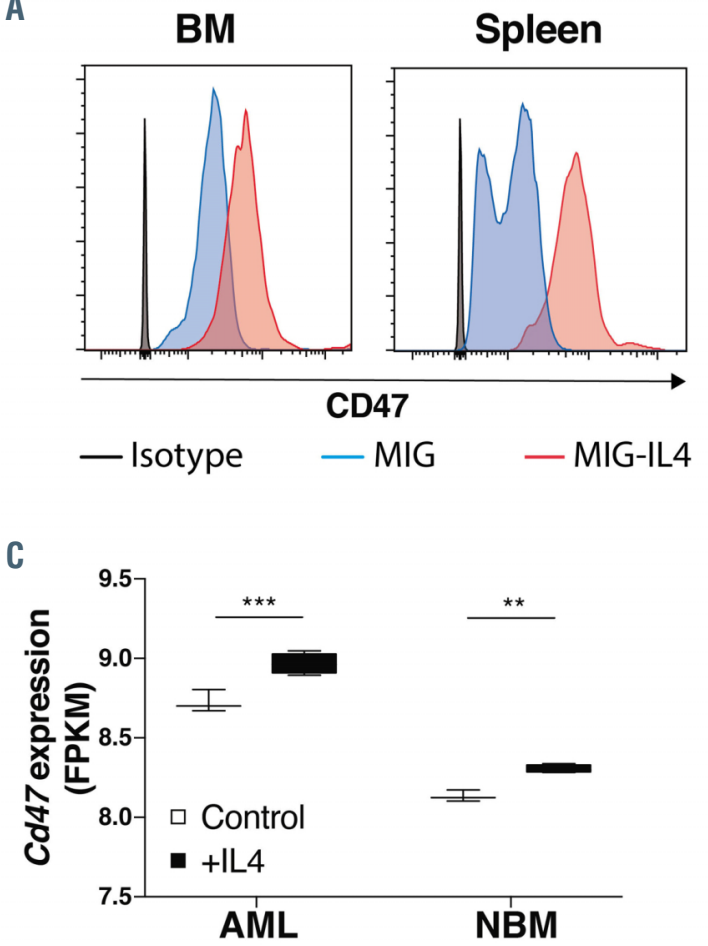

E Phagocytosis assay

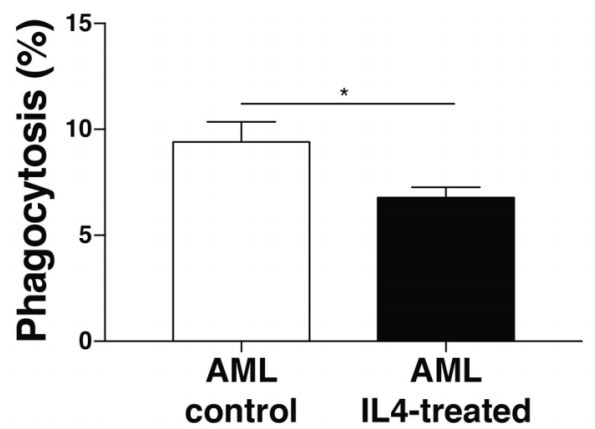

\section{Discussion}

Distinct types of macrophages control tumor development. Whereas tumor-associated macrophages promote tumor development by suppressing the immune system, other types of macrophages achieve tumor immune surveillance through phagocytosis of malignant cells. ${ }^{26-29} \mathrm{We}$ found that IL4 has antileukemic effects in mice, predominantly mediated by alternatively activated macrophages that normally play a key role in tissue repair and immune
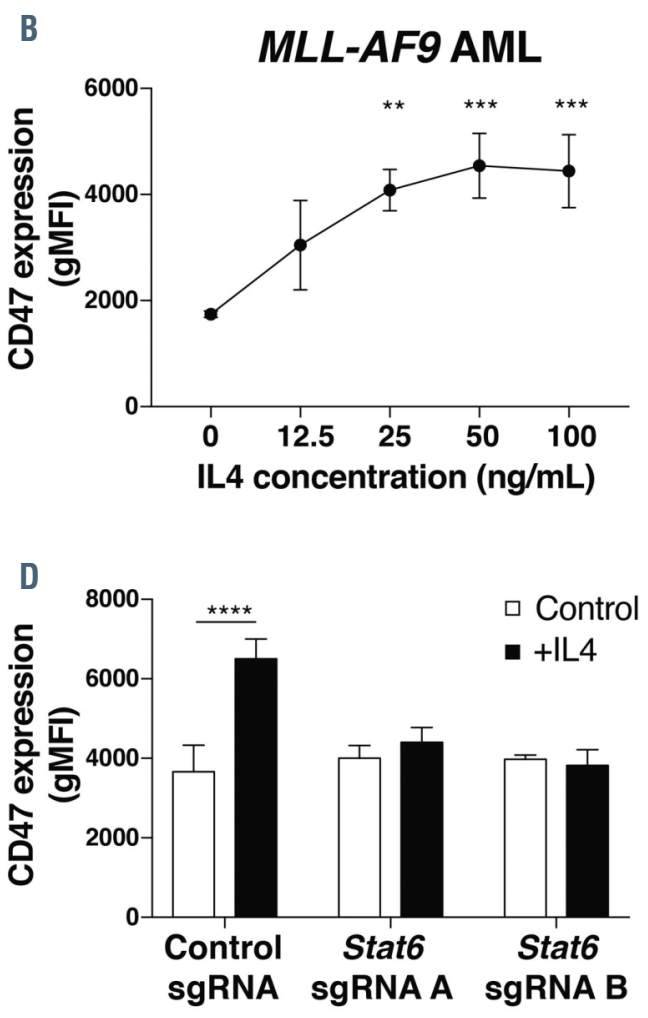

F CD47 blockade

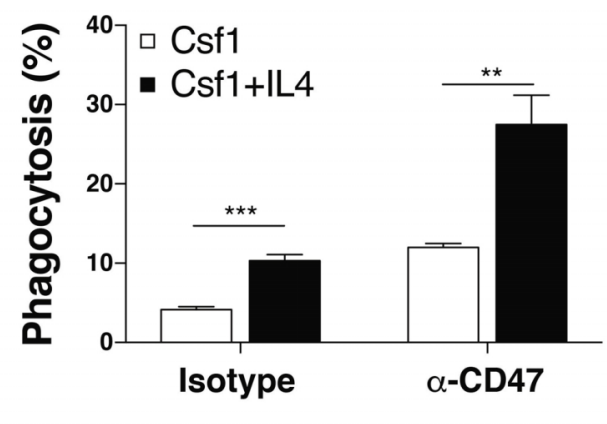

Figure 5. Combined interleukin 4 stimulation and CD47 blockade result in enhanced macrophage-mediated phagocytosis of acute myeloid leukemia cells. (A) Representative histograms showing CD47 expression on acute myeloid leukemia (AML) cells in bone marrow (BM) and spleens of mice transplanted with dsRed leukemia cells transduced with the MIG-interleukin 4 (MIG-IL4) or control (MIG) vectors. (B) CD47 expression on AML cells following IL4 stimulation for $24 \mathrm{~h}$. (C) Cd47 expression shown as FPKM values of normalized reads from RNA sequencing data of c-Kit ${ }^{+}$dsRed $^{+}$leukemia cells and c-Kit ${ }^{+}$normal BM cells stimulated with IL4 for $18 \mathrm{~h}$. Data are presented as box and whiskers diagrams; the line indicates median, box limits are first and third quartiles, and bars indicate maximum and minimum values. (D) CD47 expression measured by flow cytometry after $24 \mathrm{~h}$ of stimulation with murine (m)IL4 (100 ng/mL) in cells transduced with lentiviral vectors expressing Stat6 or control sgRNA. (E) Phagocytosis assay with macrophages derived from murine BM monocytes stimulated with mCSF1 (25 ng/mL) and mIL4 (20 $\mathrm{ng} / \mathrm{mL})$ for 7 days. The AML cells were treated with $\mathrm{mlL} 4(100 \mathrm{ng} / \mathrm{mL})$ or no IL4 (control) for $24 \mathrm{~h}$ prior to co-culture ( $\mathrm{n}=3$ ). Phagocytosis is presented as the percentage of dsRed ${ }^{+}$cells within F4/80 cells. (F) Phagocytosis assay with mouse BM monocyte-derived macrophages stimulated for 7 days with mCSF1 (25 ng/mL) and mIL4 $(20 \mathrm{ng} / \mathrm{mL})$ or mCSF1 only $(\mathrm{n}=3)$. AML cells were cultured for $1 \mathrm{~h}$ with a blocking anti-CD47 antibody or corresponding isotype control and then mixed with the macrophages. FPKM, fragments per kilobase million; gMFI, geometric mean fluorescence intensity; NBM, normal bone marrow. $* P<0.05$; $* * P<0.01 ; * \star * P<0.001$; $* * * * P<0.0001$ 
regulation. ${ }^{30,31}$ The observed expansion of alternatively activated macrophages is consistent with findings showing that IL4, via the IL 4 receptor type I complex, promotes the outgrowth of macrophages beyond homeostatic levels in the setting of nematode infections. ${ }^{32}$ However, nematode infections trigger the expansion of tissue resident macrophages. ${ }^{32}$ In contrast, the IL4-induced macrophages with antileukemic activity showed higher expression of Ccl24, Mrc1, and Pdcd1lg2, suggesting that they are of monocytic origin, from either the bone marrow or peripheral blood. ${ }^{24}$ Among hematopoietic cells, only macrophages showed increased numbers following enforced expression of IL4 in vivo. IL4 also boosted the phagocytic activity of murine monocyte-derived macrophages in vitro, suggesting that IL4 acts directly on the monocytes/macrophages that mediate the antileukemic effect. Moreover, consistent with their increased phagocytic activity, the IL4-induced macrophages were functionally and molecularly distinct from tumor-associated macrophages, which are classically associated with an alternatively activated phenotype. ${ }^{25}$ Furthermore, the IL4-induced macrophages were functionally distinct from AML-associated macrophages, which polarize into a leukemia-supportive state that accelerates disease development. ${ }^{3}$ The reason why IL4 induced stronger macrophage activation in vivo than in vitro could be related to interactions with other immune cells or the AML blasts, resulting in enhanced phagocytic activity. Of note, the macrophages were dependent on IL4 for their anti-leukemic activity as depletion of macrophages in the MIG control group did not affect the leukemia burden.

Constitutive expression of IL4 in mice has not been linked previously to anti-cancer activity, but it has been associated with excessive phagocytosis resulting in decreased blood cell counts, extramedullary hematopoiesis, and increased mortality. ${ }^{33,34}$ We found that IL4 induced potent antileukemic activity, with some mice surviving long-term without signs of disease or problems of tolerability, while other mice eventually had to be sacrificed despite very low levels of leukemia cells in their bone marrow and spleen. The low blood cell counts and expansion of megakaryocytes in the spleen indicated extramedullary hematopoiesis and suggests that elevated IL4 levels induced macrophage activation with excessive phagocytosis. This pattern resembles that of hemophagocytic lymphohistiocytosis (HLH), a disease characterized by aberrantly activated macrophages. ${ }^{35}$ Hence, we speculate that the cause of death of non-leukemic mice in the IL4 group was due to the HLH-like symptoms. Of note, the leukemic cells were selectively depleted, indicating that the IL4-induced macrophages preferentially attacked them. The reason is unclear but could be related to altered expression of genes by leukemia cells that regulate macrophages, such as MHC class I molecules or calreticulin. $^{36,37}$

In addition to IL4 boosting macrophage-mediated phagocytosis, stimulation of AML cells with IL4 induced STAT6-dependent upregulation of CD47, revealing a pre- viously unrecognized mechanism that regulates CD47 expression and thereby protects cells from phagocytosis. This mechanism could possibly have evolved to protect endogenous cells from phagocytosis in areas in which high IL4 levels activate macrophages to fight invading pathogens. Consistent with these findings, a superenhancer region with binding sites for STAT6 has been shown to regulate CD47 expression, ${ }^{38}$ providing a putative mechanistic basis for how CD47 is upregulated via the IL4/STAT6 pathway. Given that combined IL4 stimulation and CD47 inhibition enhanced macrophage-mediated phagocytosis of AML cells, our data suggest therapeutic potential for strategies that combine direct activation of macrophages with blocking of inhibitory signals to macrophages. Because IL 4 has opposing effects in murine and human macrophages, we speculate that other cytokines that activate human macrophages may also upregulate CD47 or other 'don't eat me' signals on target cells. Identifying these mechanisms may translate into new therapeutic opportunities in AML and possibly other types of cancer.

In summary, here we show that IL4 has a potent in vivo antileukemic effect in mice by promoting macrophagemediated phagocytosis of AML cells. IL4 stimulation induced CD47 upregulation in a STAT6-dependent manner, and combined IL4 stimulation with CD47 blockade further enhanced macrophage-mediated phagocytosis of AML cells. These findings deepen our understanding of how IL4 regulates murine macrophages and suggest that strategies to combine macrophage activation with CD47 inhibition should be explored further as a therapeutic approach in cancer.

\section{Disclosures}

No conflicts of interest to disclose.

\section{Contributions}

$P P M, R R, C H$ and $C J$ performed research, PPM and MJ analyzed data and wrote the manuscript, and all other authors contributed with valuable comments.

\section{Acknowledgments}

The authors thank Dr Benjamin Ebert (Brigham and Women's Hospital, Boston, MA, USA) for sharing the dsRed+MLL-AF9 leukemia cells. We also thank Dr James Mulloy, (University of Cincinnati, Cincinnati, OH, USA) for sharing the MA9:16 cells.

\section{Funding}

We thank the following granting agencies for their support: the Swedish Cancer Society, the Swedish Childhood Cancer Foundation, the Swedish Research Council, the Crafoord Foundation, the Royal Physiographic Society in Lund, and the Medical Faculty of Lund University.

\section{Data-sharing statement}

Raw data and normalized gene expression data are available in the Gene Expression Omnibus database under accession number GSE155048.

\section{References}

1. Costello RT, Sivori S, Marcenaro E, et al. Defective expression and function of natural killer cell-triggering receptors in patients with acute myeloid leukemia. Blood.
2002;99(10):3661-3667

2. Jaiswal S, Jamieson $\mathrm{CH}$, Pang WW, et al. CD47 is upregulated on circulating hematopoietic stem cells and leukemia cells to avoid phagocytosis. Cell. 2009; 138(2):271-285.
3. Al-Matary YS, Botezatu L, Opalka B, et al Acute myeloid leukemia cells polarize macrophages towards a leukemia supporting state in a growth factor independence 1 dependent manner. Haematologica. 2016; 101(10):1216-1227. 
P. Peña-Martinez et al.

4. Carlsten M, Järås M. Natural killer cells in myeloid malignancies: immune surveillance, NK cell dysfunction, and pharmacological opportunities to bolster the endogenous NK cells. Front Immunol. 2019; 10:2357.

5. Paczulla AM, Rothfelder K, Raffel S, et al. Absence of NKG2D ligands defines leukaemia stem cells and mediates their immune evasion. Nature. 2019; 572(7768): 254-259.

6. Petty AJ, Yang Y. Tumor-associated macrophages in hematologic malignancies: new insights and targeted therapies. Cells. 2019;8(12):1526.

7. Li Y, You MJ, Yang Y, Mu D, Than C. The role of tumor-associated macrophages in leukemia. Act Haematol. 2020;143(2):112117.

8. Wrangle JM, Patterson A, Johnson CB, et al. IL-2 and beyond in cancer immunotherapy. J Interferon Cytokine Res. 2018;38(2):45-68.

9. Majeti R, Cha MP, Alizadeh AA, et al. CD47 is an adverse prognostic factor and therapeutic antibody target on human acute myeloid leukemia stem cells. Cell. 2009;138(2):286-299.

10. Peña-Martínez P, Eriksson $M$, Ramakrishna $\mathrm{R}$, et al. Interleukin 4 induces apoptosis of acute myeloid leukemia cells in a Stat6-dependent mannor. Leukemia. 2018;32(3):588-596.

11. Li Z, Chen L, Ain Z. Paradoxical roles of IL4 in tumor immunity. Cell Mol Immunol. 2009;6(6):415-422.

12. Kiniwa T, Enomoto Y, Terazawa N, et al. NK cells activated by Interleukin -4 in coopration with Interleukin-15 exhibit distinctive characteristics. Proc Natl Acad Sci U S A. 2016;113(36):10139-10144.

13. Paul WE. History of interleukin-4. Cytokines. 2015;75(1):3-7.

14. Krivtsov AV, Twomey D, Fen Z, et al. Transformation from committed progenitor to leukaemia stem cell initiated by MLL-AF9. Nature. 2006;442(7104):818-822. 15. Eriksson M, Peña-Martínez P, Ramakrishna R, et al. Agonistic targeting of TLR1/TLR2 induces p38 MAPK-dependent apoptosis and NFKB-dependent differentiation of AML cells. Blood Adv. 2017;1(23):2046-2057.
16. Miller PG, Al-Shahrour F, Hartwell KA, et al. In vino RNA screening identifies a leukemia-specific dependence on integrin beta 3 signaling. Cancer Cell. 2013; 24(1):45-58.

17. Järås M, Miller PG, Chu LP, et al. Csnk1a1 inhibition has p53-dependent therapeutic efficacy in acute myeloid leukemia. J Exp Med. 2014;211(4):605-612.

18. Piganelli JD, Martin T, Haskins K. Splenic macrophages from the NOD mouse are defective in the ability to present antigen. Diabetes. 1998;47(8):1212-1218.

19. Ito $M$, Hiramatsu $H$, Kobayashi $K$, et al. $\mathrm{NOD} / \mathrm{SCID} / \gamma$ cull mouse: an excellent recipient mouse model for engraftment of human cells. Blood. 2002;100(9):3175.

20. Claassen I, Van Rooijen N, Claassen E. A new method for removal of mononuclear phagocytes from heterogeneous cell poplations in vito, using the liposome-mediated macrophage 'suicide' technique. J Immunol Methods. 1990;134(2):153-161.

21. Dian Q, Jutila MA, Van Rooijen N, Cutler JE. Elimination of mouse splenic macrophages correlates with increased sisceptibility to experimental disseminated candidiasis. J Immunol. 1994;152(10):50005008.

22. Loke Pr, Nair MG, Parkinson J, Guiliano D, Blaxter M, Allen JE. IL-4 dependent alternatively-activated macrophages have a distinctive in viva gene expression phenotype. BMC Immunol. 2002;3:7.

23. Martinez FO, Helming L, Milder R, et al. Genetic programs expressed in resting and IL -4 alternatively activated mouse and human macrophages: similarities and defferences. Blood. 2013;121(9):e57-e69.

24. Gundra UM, Girgis NM, Ruckerl D, et al. Alternatively activated macrophages derived from monocytes and tissue macrophages are phenotypically and functionally distinct. Blood. 2014;123(20):e110.

25. Haas L, Obenauf AC. Allies or enemies-the multifaceted role of myeloid cells in the tumor microenvironment. Front Immunol. 2019;10:2746.

26. Chen Y, Zhang X. Pivotal regulators of thissue homeostasis and cancer: macrophages. Exp Hematol Oncol. 2017;6:23.

27. Fens M, Chen JY, Weissman-Tsukamoto R, et al. Macrophages eat cancer cells using their own calreticulin as a guide: roles of TLR and Btk. Proc Natl Acad Sci U S A. 2015;112(7):2145-2150

28. Jaiswal S, Cha MP, Majeti R, Weissman IL. Macrophages as mediators of tumor immunosurveillance. Trends Immunol. 2010;31(6):212-219.

29. Loyher P-L, Hamon P, Laviron M, et al. Macrophages of distinct origins contribute to tumor development in the lung. J Exp Med. 2018;215(10):2536-2553.

30. Gordon S, Martinez FO. Alternative activetimon of macrophages: mechanism and funcions. Immunity. 2010;32(5):593-604.

31. Sick A, Mantovani A. Macrophage plasticity and polarization: in vive veritas. J Chin Invest. 2012;122(3):787-795.

32. Jenkins SJ, Ruckerl D, Thomas GD, et al. IL-4 directly signals tissue-resident macrophages to proliferate beyond homeostatic levels controlled by CSF-1. J Exp Med. 2013;210(11):2477.

33. Erb KJ, Rüger B, won Brevern M, Ryffel B, Schimpl A, Rivet K. Constitutive expression of interleukin (IL)-4 in vive causes autoimmune-type disorders in mice. J Exp Med. 1997;185(2):329-339.

34. Miler JD, Orekov T, Ward JM, et al. Sustained IL-4 exposure leads to a novel pathway for hemophagocytosis, inflammaion, and tissue macrophage accumulation. Blood. 2010;116(14):2476-2483.

35. La Rosée P, Horne A, Hines $M$, et al. Recommendations for the management of hemophagocytic lymphohistiocytosis in adults. Blood. 2019;133(23):2465-2477.

36. Barkal AA, Weiskopf K, Ka KS, et al. Engagement of MHC class I by the inhibitory receptor LILRB1 suppresses macrophages and is a target of cancer immunotherapy. Nat Immunol. 2018; 19(1):76-84.

37. Fens M, Marion KD, Zhu F, et al. Programmed cell removal by calreticulin in tissue homeostasis and cancer. Nat Common. 2018;9(1):3194.

38. Betancur PA, Abraham BJ, Yiu YY, et al. A CD47-associated super-enhancer links proinflammatory signalling to CD47 upregulaion in breast cancer. Nat Commune. 2017; 8:14802.

824

haematological | 2022; 107(4) 